\title{
Prospective evaluation of EUS-guided fine needle biopsy in pancreatic mass lesions
}

\section{(ㄷ)(이 $\odot$}

Authors

M.H. Larsen ${ }^{1}$, C.W. Fristrup ${ }^{1}$, S. Detlefsen ${ }^{2}$, M.B. Mortensen ${ }^{1}$

Institutions

1 Odense Pancreas Center (OPAC), Department of Surgery, Odense University Hospital, Odense C, Denmark

2 Odense Pancreas Center (OPAC), Department of Pathology, Odense University Hospital, Odense C, Denmark

submitted 2.7.2017

accepted after revision 20.10.2017

\author{
Bibliography \\ DOI https://doi.org/10.1055/s-0043-124078 | \\ Endoscopy International Open 2018; 06: E242-E248 \\ (c) Georg Thieme Verlag KG Stuttgart · New York \\ ISSN 2364-3722
}

Corresponding author

Michael H. Larsen, Odense Pancreas Center (OPAC), Department of Surgery, Odense University Hospital, Sdr. Boulevard 29, 5000 Odense C, Denmark

Fax: +004565412219

Michael.Hareskov.Larsen@rsyd.dk

\section{ABSTRACT}

Background and study aim Due to the scarcity of specific data on endoscopic ultrasound (EUS)-guided fine-needle biopsies (SharkCore) FNB in the evaluation of pancreatic le- sions, we performed a prospective study of the diagnostic performance of EUS SharkCore FNB in patients with pancreatic lesions. The aim of this study was to evaluate the diagnostic accuracy.

Patients and methods Single-center prospective study of 41 consecutive patients referred for EUS-FNB from October 2015 to April 2016 at our center. EUS-FNB was obtained in a predefined setting regarding the procedure and pathological evaluation. Data regarding demographics, lesion, technical parameters, and diagnostic accuracy were obtained.

Results The study included 41 consecutive patients (22 males (54\%); median age 68 years). The average size of the lesions was $28 \mathrm{~mm}$ (median: $30 \mathrm{~mm}$ ). A diagnostic specimen was identified in 40 (98\%) cases during microscopy with an average of 2.4 passes. The route was trans-duodenal in 20 cases (49\%). The histological diagnosis of the specimens was malignant in 29 cases $(71 \%)$, benign in $8(20 \%)$, suspicious in $2(5 \%)$, atypical in $1(2 \%)$ and in $1(2 \%)$ no material for microscopic evaluation was obtained. This led to a diagnostic accuracy of $93 \%$, sensitivity of $91 \%$ and a specificity of $100 \% .2$ cases (5\%) of self-limiting bleeding were observed. The diagnosis at follow up was malignant in 32 (78\%) of the patients.

Conclusions EUS-FNB of pancreatic mass lesions with the SharkCore needle produced specimens with a diagnostic accuracy of $93 \%$. The procedure was safe and easy to perform, and these data support the use of EUS-FNB in a routine setting.

\section{Introduction}

Endoscopic ultrasound -guided fine-needle aspiration (EUSFNA) has for many years been recommended when evaluating mass lesions of the pancreas where microscopic analysis was deemed necessary for a clear-cut diagnosis [1]. FNA allows evaluation of cytological features suggestive or diagnostic of malignancy with high sensitivity and specificity, and the diagnostic accuracy is even higher when an on-site pathologist is present to evaluate the quality of the obtained material [2 - 5]. However, due to lack of resources, this scenario is not possible in many centers $[3,4]$. FNAs are also faced with other problems.
Chronic pancreatitis can cause atypical cellular changes making the distinction from well-differentiated neoplasia based solely on cytological criteria difficult. In such difficult cases, the possibility to cut additional sections from a formalin-fixed and paraffin embedded tissue block with application of immunohistochemical markers may increase the diagnostic accuracy. Moreover, some entities such as mesenchymal tumors, autoimmune pancreatitis (AIP), lymphoma [6] and a few rare pancreatic primary tumors are difficult to assess by FNA. Such lesions are therefore better analyzed based on EUS-guided fine needle biopsies (FNB) or tissue fragments obtained by EUS-FNA also 
making it possible to perform molecular and genetic analyses for personalized treatment [7-9].

Since 2002 it has been possible to perform core biopsy guided by EUS using the so-called Quick-Core needle (Cook Endoscopy Inc, Limerick, Ireland), and more recently the ProCore needle from the same manufacturer has been evaluated [10, 11]. Currently, there is no standard regarding needle type, size, or biopsy technique when performing pancreatic EUSFNB. A recently published meta-analysis comparing ProCore FNB and standard FNA did not demonstrate a significant difference regarding sample adequacy, accuracy or acquisition of histological material for evaluation. The ProCore needle, however, established the diagnosis with significant fewer passes [12]. Experience with a novel FNB needle (SharkCore FNB needle, Medtronic, Dublin, Ireland) demonstrated excellent pathologic diagnostic performance also with a minimum of passes $[13,14]$.

Due to the lack of specific data on EUS SharkCore FNB in the evaluation of pancreatic lesions, we performed a prospective study of the diagnostic performance of EUS SharkCore FNB in patients with pancreatic mass-forming lesions. The aim of this study was to evaluate the diagnostic accuracy of the needle. EUS-FNB was only performed if it would have impact on the treatment strategy [15].

\section{Patients and methods}

Patients referred for EUS-FNB from October 1, 2015 to April 30, 2016 were included in this consecutive and prospective singlecenter trial, until 40 patients had histological or sufficient cytological material for microscopic evaluation. The center is a high-volume center performing around 1000 EUS procedures including 350 tissues samples a year (combination of FNA and FNB). All the participating endoscopists were experienced and had performed more than 150 procedures including tissue sampling prior to start of this study. Inclusion criteria were presence of a solid pancreatic mass on computed tomography (CT) and/or magnetic resonance imaging and/or EUS, clinical need for an exact diagnosis, age $>18$ years, and ability to provide informed consent. After EUS-FNB the final diagnosis (benign/malignant) was established using 1 of the following methods: (1) definite proof of malignancy on a surgical specimen or biopsy of a metastatic lesion; (2) malignant diagnosis on EUSFNB and clinical/imaging follow-up compatible with malignant disease; or (3) no proof of malignancy on neither EUS-FNB nor during clinical/imaging follow-up for at least 6 months. Patients with a resectable mass on EUS and/or CT went directly for surgery. This study concerns patients with a suspected but doubtful or non-resectable mass. Non-resectable masses were biopsied for final diagnosis before oncological treatment. Complications were identified by retrospective review of the electronic health records after at least 6 months both in house and in the referring hospitals.

The study was approved by The Regional Committees on Health Research Ethics for Southern Denmark and registered in the ClinicalTrial.gov database (NCT03016637). Written and informed consent was obtained from all enrolled patients.

\section{EUS-FNB}

EUS-FNB was only performed if the result would have impact on the treatment strategy, i. e. if there was a clinical need for an exact diagnosis of the histological type of pancreatic mass lesion. EUS-FNB was performed using the SharkCore needle, guided by a convex array echo-endoscope (Pentax EG-3870UTK; Pentax Europe, GmbH, Hamburg, Germany). The endoscopist decided whether to use the 25-G or the 22-G needle, and the pancreatic mass lesion was punctured using the trans-duodenal or transgastric approach. Prior to puncturing the target lesion, the stylet was retracted a few millimeters, and the tip of the needle was advanced into the target tissue under endosonographic guidance. The stylet was then retracted, while multiple movements of the needle within the lesion were performed (slow pull technique) [16]. If the second pass did not result in macroscopically visible core fragments as judged by the endoscopist, suction was applied. Suction was released before removal of the needle from the target. 1 to 3 needle passes were performed. Tissue was recovered in formalin by pushing air and eventually the stylet through the needle. All samples were processed at the Department of Pathology, Odense University Hospital (OUH). No pathologist was present in the endoscopy room.

\section{Specimen preparation and evaluation}

The SharkCore biopsies were fixed in formalin ( $6-24$ hours) end embedded in paraffin. Thirteen serial sections were cut from the paraffin embedded tissue blocks. Section no. 1 and 13 were stained with hematoxylin \& eosin (H\&E), while section no. 2 was stained with Alcian blue periodic acid-Schiff (AbPAS $\mathrm{pH}$ 2.7). Sections 3-12 were initially left unstained. If necessary for the final diagnosis, some of these sections were immunohistochemically stained for markers such as CD56, monoclonal CEA, chromogranin A, CK7, CK8, CK20, IMP3, Ki67, maspin, S100P, SMAD4, synaptophysin or VHL. Additional markers were used when necessary. All cases were evaluated and signed out by 1 of 4 pathologists specialized in gastrointestinal pathology.

\section{Statistics}

Continuous data are presented with mean and standard deviation. Results of diagnostic tests are reported with $95 \%$ confidence intervals. Stata v. 12 (StataCorp, USA) was used.

\section{Results}

The study included 41 consecutive patients, 22 males (54\%) and 19 females (46\%), with an average age of 68 years (range $36-88) .91 \%$ of the patients were in WHO performance status $0-1.40$ patients ( $98 \%$ ) had a CT performed prior to EUS evaluation and EUS-FNB ( $\triangleright$ Table1). The final diagnosis was malignant in $32(78 \%)$ patients.

\section{EUS-FNB procedure}

7 endosonographers performed the 41 EUS-FNBs included in this study. The 22-G needle was used in 38 (93\%) cases and the $25 \mathrm{G}$ needle in 3 (7\%). An average of 2.4 passes was used (range $1-3$ ), and the biopsies were obtained by the slow pull- 


\begin{tabular}{|l|c|}
\hline Table 1 Clinical data on 41 EUS-FNB patients. \\
\hline Gender & $19(46 \%)$ \\
\hline Female & $22(54 \%)$ \\
\hline Male & \\
\hline Age & $68 \pm 11$ years \\
\hline Average & $36-88$ years \\
\hline Range & \\
\hline WHO Performance Status & $13(32 \%)$ \\
\hline 0 & $24(59 \%)$ \\
\hline 1 & $3(7 \%)$ \\
\hline 2 & $1(2 \%)$ \\
\hline 3 & \\
\hline Diagnostic workup before EUS-FNB & $40(98 \%)$ \\
\hline CT & $2(5 \%)$ \\
\hline PET-CT & $5(12 \%)$ \\
\hline ERCP & $32(78 \%)$ \\
\hline Final diagnosis & $9(22 \%)$ \\
\hline Malignant & $7(17 \%)$ \\
\hline Benign & \\
\hline Final diagnosis based on & \\
\hline Histology-FNB & \\
\hline Histology-Surgery & \\
\hline Follow-up & \\
\hline
\end{tabular}

technique in 39 (95\%) cases. The average size of the pancreatic lesions was $28 \pm 11 \mathrm{~mm}$ (median: $30 \mathrm{~mm}$ ). The access route was trans-duodenal in 20 cases (49\%) and trans-gastric in 21 (51\%) cases. 2 cases ( $5 \%$ ) of self-limited bleeding were observed during the biopsy procedure, but none of the patients required additional treatment or follow-up. There were not registered any additional complications requiring contact to our institution or the referring hospital. The endosonographers identified and documented tissue cores or fragments in all patients.

\section{Microscopic evaluation of the EUS-FNBs}

A diagnostic specimen was identified in 40 (98\%) cases during microscopy, and the quality of the obtained biopsy was considered "good" in 39 (95\%) cases. Details of the microscopic examination are shown in $>$ Table 2 . Immunohistochemistry was used in 38 (93\%) of the cases as a supplement to H\&E and AbPAS. Histological diagnosis of EUS-FNB specimens was malignant in 29 cases (71\%) and benign in 8 cases (20\%). 2 (5\%) specimens were considered suspicious of malignancy, whereas 1 only consisted of a cytological smear of atypical cells (2\%) and another (2\%) contained several blood cylinders but no material relevant for microscopic evaluation. Among the malignant tumors, $90 \%$ were classified as ductal adenocarcinomas of the pancreas ( $\triangleright$ Fig.1a and $\triangleright$ Fig.1b) and the remaining $10 \%$ as
- Table 2 Histological evaluation of EUS-FNB.

Quality of specimen as assessed by the pathologist $(n=41)$

Good $39(95 \%)$

Poor

$1(2 \%)$

No diagnostically useful specimen obtained

$1(2 \%)$

FNB histological diagnosis

Malignant

$29(71 \%)$

- Adenocarcinoma $26(90 \%)$

- NET

$3(10 \%)$

Suspicion of malignancy $2(5 \%)$

- IPMN $1(50 \%)$

- High-grade dysplasia $1(50 \%)$

Atypical cells $1(2 \%)$

- Fibrosis

$1(100 \%)$

Benign $8(20 \%)$

- Pancreatitis $2(25 \%)$

- Fibrosis $2(25 \%)$

- IPMN $2(25 \%)$

- Suspicion of leiomyomatous tumor $1(12.5 \%)$

- Normal tissue $1(12.5 \%)$

No diagnostically specimen obtained (blood cylinders)

$1(2 \%)$

Gold standard / final histologic diagnosis

\begin{tabular}{|l|c|}
\hline Adenocarcinoma & $29(71 \%)$ \\
\hline NET & $3(7 \%)$ \\
\hline Benign lesion & $9(22 \%)$ \\
\hline
\end{tabular}

FNB, fine-need biopsy; IPMN, intraductal papillary mucinous neoplasm; NET, neuroendocrine tumor

neuroendocrine tumors (NETs) ( $>$ Fig. 1c and $>$ Fig. 1 d). 3 specimens were indicative of an intraductal-papillary mucinous neoplasm (IPMN) ( $\triangleright$ Fig. 1e and $\triangleright$ Fig. 1f). 1 of the IPMN cases was also suspicious of malignancy ( $>$ Table 2 ).

\section{Follow-up}

None of the cases with a benign histological diagnosis turned out to be malignant after at least 6 months of follow-up with clinical evaluation and imaging. The patient with atypical cells had a histologically confirmed malignant tumor, detected at follow-up by laparoscopic ultrasound (LUS) guided core needle biopsy. Of the 2 patients in whom malignancy was suspected, 1 had a malignant tumor diagnosed by a second SharkCore biopsy. In the calculation of the sensitivity, only conclusive malignant histological diagnoses were considered malignant, whereas cases specified as "atypical cells" and "suspicious of malignancy" were considered benign. This led to a sensitivity of $91 \%$ and a specificity of $100 \%$, and an overall accuracy of $93 \%$ ( $\triangleright$ Table 3 ). There were no false-positive and 3 (7\%) false- 


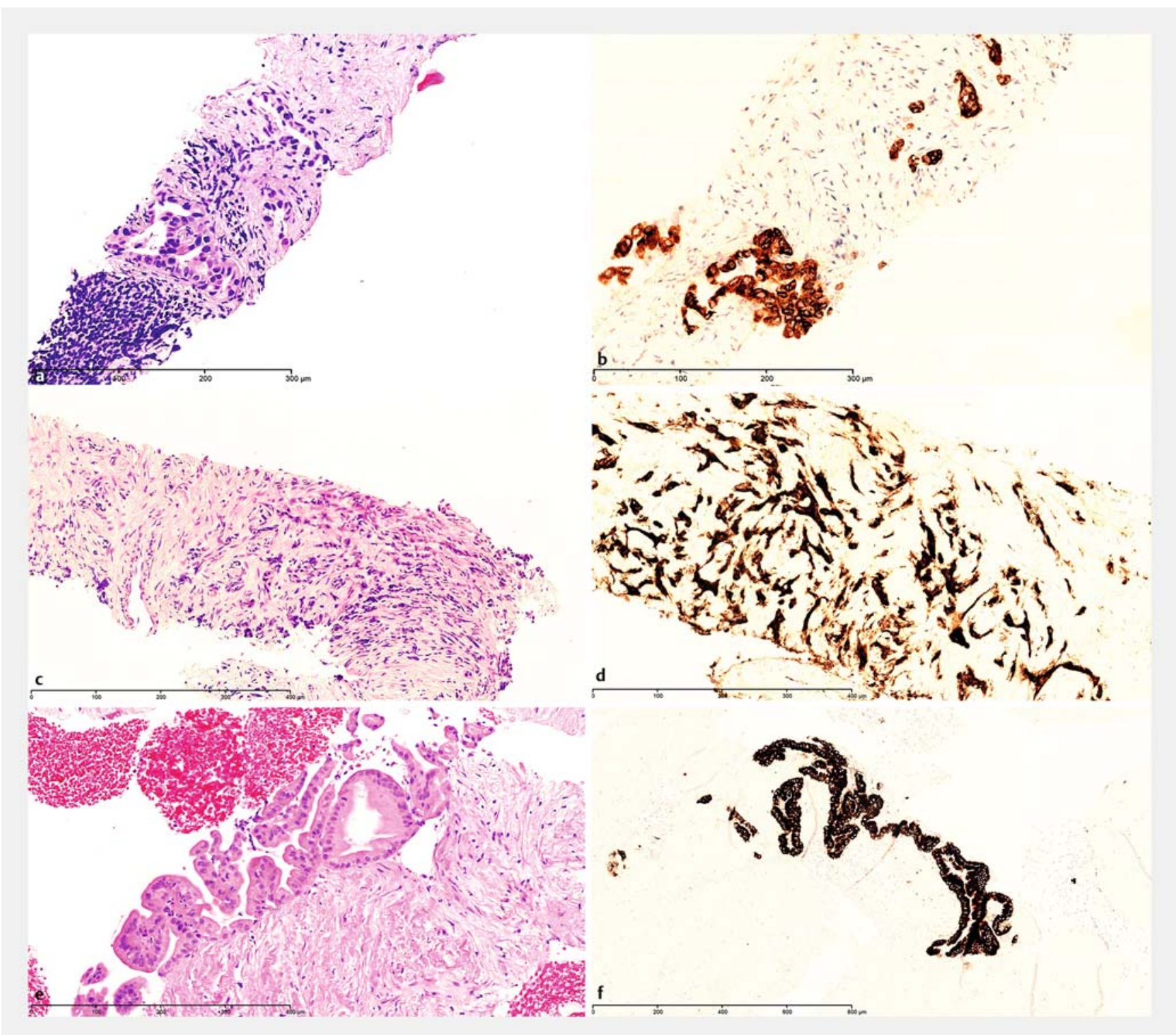

- Fig. 1 Microscopic examples of EUS-guided SharkCore biopsies from the pancreas. a H\&E and b IMP3 immunostaining of ductal adenocarcinoma of the pancreas. $\mathbf{c H \& E}$ and $\mathbf{d}$ insulin immunostaining of insulin-producing neuroendocrine tumor of the pancreas. e H\&E and $\mathbf{f} M U C 5 \mathrm{AC}$ immunostaining of intraductal-papillary mucinous neoplasm (IPMN) with gastric-type epithelium, low-grade dysplasia.

negative specimens observed in this study. The overall correlation between EUS-FNB and gold standard is shown in > Fig. 2. Follow up for non-malignant cases is shown in $>$ Table 4.

\section{Discussion}

EUS plays a central role in evaluation of pancreatic masses, and if cytological proof of malignancy is clinically relevant for optimal decision-making, cytological specimens can be obtained using EUS-FNA. Test performance of standard EUS-FNA needles has been evaluated in several prospective studies on patients with solid pancreatic mass lesions. A meta-analysis demonstrated a pooled sensitivity for EUS-FNA of $86.8 \%$ (95\% confidence interval $[\mathrm{Cl}], 85.5-87.9)$ and a pooled specificity of $95.8 \%(95 \% \mathrm{Cl}, 94.6-96.7)$ [17]. Despite these data the diag-
- Table 3 Diagnostic accuracy of EUS-FNB using the Sharkcore needle. Intention to treat $-\mathrm{N}=41$

\begin{tabular}{|l|l|}
\hline Sensitivity & $0.91(0.75-0.98)$ \\
\hline Specificity & $1.00(0.66-1)$ \\
\hline Positive predictive value & $1.00(0.88-1)$ \\
\hline Negative predictive value & $0.75(0.43-0.95)$ \\
\hline Overall accuracy & $93 \%(80-98 \%)$ \\
\hline
\end{tabular}

( $95 \%$ confidence intervals); EUS-FNB, endoscopic ultrasound-guided fineneed biopsy 


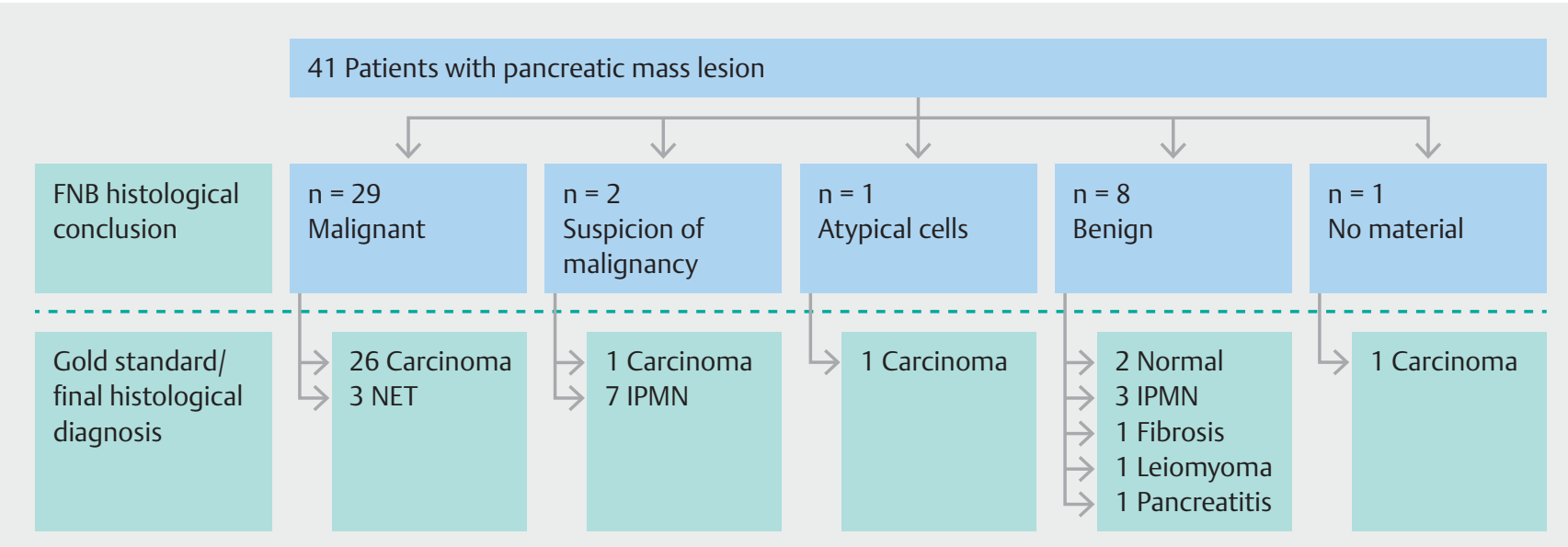

- Fig. 2 Flowchart of patients undergoing EUS-FNB for pancreatic mass lesions. Blue: FNB Histological conclusion. White: Gold standard (final histological diagnosis/follow up)

- Table 4 Follow up of non-malignant cases plus number of procedures during the follow-up period of 6 months.

\begin{tabular}{|c|c|c|c|c|c|}
\hline Patient number & FNB & EUS + Biopsy & LUS + Biopsy & Ст & $\begin{array}{l}\text { Final } \\
\text { diagnosis }\end{array}$ \\
\hline 4 & Benign (suspicion of leiomyomatous tumor) & - & + & + & Leiomyoma \\
\hline 5 & Benign (IPMN with LGD) & ++ & - & ++ & IPMN - LGD \\
\hline 6 & Benign (pancreatitis) & - & - & + & Pancreatitis \\
\hline 13 & Benign (pancreatitis) & + & + & + & Pancreatitis \\
\hline 15 & Atypical (fibrosis) & - & + & - & Adenocarcinoma \\
\hline 16 & Non-tissue (blood clots) & + & - & - & Adenocarcinoma \\
\hline $19^{1}$ & Benign (fibrosis) & + & - & ++ & PANIN-2 \\
\hline 25 & Suspicion of malignancy (HGD) & + & - & - & Adenocarcinoma \\
\hline 28 & Benign (fibrosis) & - & - & + & Pancreatitis \\
\hline 34 & Suspicion of malignancy (IPMN) & ++ & - & ++ & PANIN \\
\hline 35 & Benign (normal tissue) & $+(-$ biopsy) & - & ++ & Normal pancreas \\
\hline 40 & Benign (IPMN with LGD) & ++ & - & ++ & IPMN - LGD \\
\hline
\end{tabular}

CT, computed tomography; EUS, endoscopic ultrasound; FNB, fine-needle biopsy; HGD, high-grade dysplasia; IPMN, intraductal papillary mucinous neoplasm; LGD, low-grade dysplasia; LUS, laparoscopic ultrasound; PAN IN, pancreatic intraepithelial neoplasia

${ }^{1}$ The surveillance was stopped after 3 months due to severer psychiatric disease.

nostic yield may vary significantly, and the EUS-FNA technique and the reported data suffer from several limitations. Endosonographer experience [18], institution volume, presence or absence of on-site pathologist $[3-5,19]$, number of passes and needles used [20], cytological rating of indeterminate diagnoses [21], presence of concomitant chronic pancreatitis, and success rates outside clinical trials are just a few of the relevant issues.

To circumvent some of these problems, several attempts have been undertaken to obtain EUS-FNB for histological analyses. Initial efforts were directed toward using large-caliber (18 G) needles [22], and the Quick-Core and the ProCore biopsy needles demonstrated diagnostic accuracy ranging from $42 \%$ to $90 \%[10,12,23,24]$. However, large-caliber needles may reduce maneuverability in the duodenum and increase the rate of complications, and focus has turned towards EUS-FNB with 25G and $22 \mathrm{G}$ needles. Preliminary retrospective data using the $25 \mathrm{G}$ and 22G SharkCore FNB needles in pancreatic lesions provided a pathological diagnostic yield of $86 \%$ to $95 \%$ with a mean number of passes of only $2[13,14]$. In addition, the SharkCore needle seemed able to provide significantly larger histological specimens than the standard EUS-FNA needle [25]. The reported lower number of passes with EUS-FNB was in concordance with a meta-analysis comparing standard FNA and ProCore FNB [12]. However, the diagnostic yield was identical between the standard FNA needle and the FNB needle, and a subset anal- 
ysis of pancreatic biopsies from a recent randomized study were unable to detect any differences in the cellularity yield, mean number of passes and reported adverse events [26].

Both EUS-FNA and FNB may sometimes fail to provide sufficient material to establish the final diagnosis. Studies suggest that EUS re-biopsy in patients with previous non-diagnostic EUS-FNA from pancreatic lesions will lead to higher accuracy rates [27], but this also means additional EUS investigations and use of needles, increasing the costs. Therefore, any new EUS-FNB needle should be monitored (and preferably tested against standard EUS-FNA needles in a randomized trial) regarding manoeuvrability, tumor access, diagnostic accuracy, number of passes, safety profile and overall costs until a final diagnosis is reached.

Using the SharkCore needle in a prospective setting, we obtained tissue suitable for microscopic analysis in the majority of cases (98\%), and the overall diagnostic accuracy for evaluating pancreatic tumors was $93 \%$. A similar high diagnostic yield of $86 \%$ to $95 \%$ and $87 \%$ for pancreatic lesions has been published using the SharkCore needle and the ProCore needle, respectively in retrospective studies [12-14]. An interesting finding in the present study was the high proportion (49\%) of FNB procedures being performed through the duodenum with the endoscope in a J-flexed position. We experienced no needle handling problems in this position. The $25-G$ needle was used in only 3 early cases ( 1 through the stomach), and after these initial experiences the $22 \mathrm{G}$ was chosen as standard for all EUS-FNB procedures. The needle flexibility and the trans-duodenal access have been reported as limitations especially with the QuickCore needle $[11,23]$ but also with the ProCore needle, where resistance to removing the stylet was hard in $18 \%$ and removal impossible in $2 \%$ [10]. We experienced no problems related to the use or removal of the stylet from the SharkCore needle in any position, but difficulty in puncturing the target was reported in 1 case. However, sufficient histological material was obtained from this lesion, leading to suspicion of a leiomyoma. In all samples a visible core was identified by the endosonographer. However, the inadequate sample consisted of several blood cylinders at microscopy. To optimize macroscopic evaluation of the specimens, we have tried photo documentation and macroscopic measuring of the tissue cores. None of the measures mentioned had a convincing effect.

A randomized study has shown the superiority of the fanning technique compared to the standard technique during EUSFNA of solid pancreatic mass lesions [28]. It is easy to apply the same technique with the SharkCore needle, but there are no such data regarding this needle and it was not specifically investigated in the present study. A mean of 2.4 passes were performed until a macroscopic specimen was obtained as judged by the endoscopist, and this correlated with a diagnostically conclusive biopsy specimen for microscopic evaluation in $95 \%$ of the cases. 1 specimen (2.5\%) contained only cytological material and 1 (2.5\%) only blood without any relevant tissue for analysis. Studies evaluating the diagnostic accuracy of the SharkCore needle have reported a higher diagnostic accuracy when comparing histological to cytological material, and in both studies a histological biopsy was obtained with a median number of 2 needle passes [13,14]. However, the potential clinical relevance of a low number of needle passes (i. e. regarding the rate of complications) is unknown.

We experienced no complications apart from 2 small selflimited bleeds during the procedure, which were asymptomatic. In the only other study reporting on EUS SharkCore FNBrelated complications, a mild pancreatitis was reported in $2.9 \%$. This is slightly higher than expected since a systematic review found procedure-related pancreatitis in $0.4 \%$ at pancreatic EUS-FNA [29]. However, larger prospective studies are needed to establish the true risk profile of the SharkCore needle.

Thus, EUS-guided biopsy plays a significant role in up to onethird of patients suspected of having pancreatic cancer, and the actual clinical impact of EUS-guided biopsy will probably increase, as more (rare) pancreatic diseases may be detected when EUS-FNA and EUS-FNB are used more frequently [15]. Based on these first prospective data, EUS-guided SharkCore biopsy may provide more material for pathological evaluation and thus make a substantial contribution to the area of minimal invasive diagnostics.

There are several limitations to this single-center non-comparative trial, and the results may not reflect practices or technologies used at other institutions. The relatively high number of malignant tumors included may have biased the results. Another critical point in the present study is the use and definition of the criterion-standard reference method. Ideally, when the EUS-FNB was negative, histological confirmation based on surgical specimens would be the criterion standard, but this cannot be obtained for ethical reasons in this type of patients. Therefore, clinical follow-up for at least 6 months with repeated imaging procedures was used in these patients. Although not ideal, this method is a well-accepted reference standard. 7 different endosonographers performed the biopsies, but we do not consider this a true limitation, but rather a reflection of every day routine in a large and busy endoscopic unit. In addition, the lack of complications and technical problems may indicate that the FNB needle is easy to use and without a learning curve.

\section{Conclusion}

In conclusion, EUS-FNB of pancreatic mass lesions with the SharkCore needle produced specimens with a diagnostic accuracy of $93 \%$. The procedure was safe and easy to perform, and these preliminary data support the use of EUS-FNB in a routine setting where establishing the histological type of pancreatic tumors is clinically relevant. Future prospective and comparative studies should focus on safety, diagnostic accuracy and costs.

\section{Competing interests}

None 


\section{References}

[1] Storm AC, Lee LS. Endoscopic ultrasound-guided techniques for diagnosing pancreatic mass lesions: Can we do better? World J Gastroenterol 2016; 22: 8658-8669

[2] Nelsen EM, Buhler D, Soni AV et al. Endoscopic ultrasound in the evaluation of pancreatic neoplasms-solid and cystic: A review. World J Gastrointest Endosc 2015; 7: 318 - 327

[3] Iglesias-Garcia J, Larino-Noia J, Abdulkader I et al. Rapid on-site evaluation of endoscopic-ultrasound-guided fine-needle aspiration diagnosis of pancreatic masses. World J Gastroenterol 2014; 20: 9451 9457

[4] Ecka RS, Sharma M. Rapid on-site evaluation of EUS-FNA by cytopathologist: an experience of a tertiary hospital. Diagn Cytopathol 2013; 41: $1075-1080$

[5] Khan MA, Grimm IS, Ali R et al. A meta-analysis of endoscopic ultrasound-fine-needle aspiration compared to endoscopic ultrasoundfine-needle biopsy: diagnostic yield and the value of onsite cytopathological assessment. Endosc Int Open 2017; 5: E363 - E375

[6] Bhutani M, Koduru P, Lanke G et al. The emerging role of endoscopic ultrasound-guided core biopsy for the evaluation of solid pancreatic masses. Minerva Gastroenterol Dietol 2015; 61: 51 - 59

[7] Brais RJ, Davies SE, O'Donovan M et al. Direct histological processing of EUS biopsies enables rapid molecular biomarker analysis for interventional pancreatic cancer trials. Pancreatology 2012; 12: 8-15

[8] Christensen L, Mortensen MB, Detlefsen S. Breast Carcinoma With Unrecognized Neuroendocrine Differentiation Metastasizing to the Pancreas: A Potential Diagnostic Pitfall. Int J Surg Pathol 2016; 24: $463-467$

[9] Cheng B, Zhang Y, Chen Q et al. Analysis of Fine-Needle Biopsy Versus Fine-Needle Aspiration in Diagnosis of Pancreatic and Abdominal Masses: A Prospective, Multicenter, Randomized Controlled Trial. Clin Gastroenterol Hepatol 2017: doi:10.1016/j.cgh.2017.07.010

[10] Iglesias-Garcia J, Poley J, Larghi A et al. Feasibility and yield of a new EUS histology needle: results from a multicenter, pooled, cohort study. Gastrointest Endosc 2011; 73: 1189-1196

[11] Levy M], Jondal ML, Clain J et al. Preliminary experience with an EUSguided trucut biopsy needle compared with EUS-guided FNA. Gastrointest Endosc 2003; 57: 101 - 106

[12] Bang JY, Hawes R, Varadarajulu S. A meta-analysis comparing ProCore and standard fine-needle aspiration needles for endoscopic ultrasound-guided tissue acquisition. Endoscopy 2016; 48: 339-349

[13] DiMaio C], Kolb JM, Benias PC et al. Initial experience with a novel EUSguided core biopsy needle (SharkCore): results of a large North American multicenter study. Endosc Int Open 2016; 4: E974 - E979

[14] Kandel P, Tranesh G, Nassar A et al. EUS-guided fine needle biopsy sampling using a novel fork-tip needle: a case-control study. Gastrointest Endosc 2016; 84: 1034 - 1039

[15] Mortensen MB, Pless T, Durup J et al. Clinical impact of endoscopic ultrasound-guided fine needle aspiration biopsy in patients with up- per gastrointestinal tract malignancies. A prospective study. Endoscopy 2001; 33: $478-483$

[16] Nakai Y, Isayama H, Chang KJ et al. Slow pull versus suction in endoscopic ultrasound-guided fine-needle aspiration of pancreatic solid masses. Dig Dis Sci 2014; 59: 1578 - 1585

[17] Puli SR, Bechtold ML, Buxbaum JL et al. How good is endoscopic ultrasound-guided fine-needle aspiration in diagnosing the correct etiology for a solid pancreatic mass?: A meta-analysis and systematic review Pancreas 2013; 42: 20-26

[18] Shahidi N, Ou G, Lam E et al. When trainees reach competency in performing endoscopic ultrasound: a systematic review. Endosc Int Open 2017; 5: E239-E243

[19] Jhala NC, Grimm IS, Ali B et al. Providing on-site diagnosis of malignancy on endoscopic-ultrasound-guided fine-needle aspirates: should it be done? Ann Diagn Pathol 2007; 11: $176-181$

[20] Erickson RA, Sayage-Rabie L, Beissner RS. Factors predicting the number of EUS-guided fine-needle passes for diagnosis of pancreatic malignancies. Gastrointest Endosc 2000; 51: $184-190$

[21] Virk RK, Gamez R, Mehrotra S et al. Variation of cytopathologists' use of the indeterminate diagnostic categories "atypical" and "suspicious for malignancy" in the cytologic diagnosis of solid pancreatic lesions on endoscopic ultrasound-guided fine-needle aspirates. Diagn Cytopathol 2017; 45: 3-13

[22] Binmoeller KF, Thul R, Rathod V et al. Endoscopic ultrasound-guided, 18-gauge, fine needle aspiration biopsy of the pancreas using a 2.8 mm channel convex array echoendoscope. Gastrointest Endosc 1998; 47: $121-127$

[23] Sakamoto H, Kitano M, Komaki T et al. Prospective comparative study of the EUS guided 25-gauge FNA needle with the 19-gauge Trucut needle and 22-gauge FNA needle in patients with solid pancreatic masses. J Gastroenterol Hepatol 2009; 24: 384-390

[24] Gerke H, Rizk MK, Vanderheyden AD et al. Randomized study comparing endoscopic ultrasound-guided Trucut biopsy and fine needle aspiration with high suction. Cytopathology 2010; 21: 44-51

[25] Jovani M, Abidi WM, Lee LS. Novel fork-tip needles versus standard needles for EUS-guided tissue acquisition from solid masses of the upper GI tract: a matched cohort study. Scand J Gastroenterol 2017; 52: $784-787$

[26] Othman MO, Abdelfatah MM, Padilla O et al. The cellularity yield of 3 different 22-gauge endoscopic ultrasound fine needle aspiration needles. Diagn Cytopathol 2017; 45: 426-432

[27] Ainsworth AP, Hansen T, Fristrup CW et al. Indications for and clinical impact of repeat endoscopic ultrasound. Scand J Gastroenterol 2010; 45: $477-482$

[28] Bang JY, Magee SH, Ramesh J et al. Randomized trial comparing fanning with standard technique for endoscopic ultrasound-guided fineneedle aspiration of solid pancreatic mass lesions. Endoscopy 2013; 45: $445-450$

[29] Wang KX, Ben QW, Jin ZD et al. Assessment of morbidity and mortality associated with EUS-guided FNA: a systematic review. Gastrointest Endosc 2011; 73: 283-290 